\title{
The Treachery of Images
}

Patrick C. Hackler, BS, Richard B. Gunderman, MD, PhD

Indiana University School of Medicine, 702 North Barnhill Drive, Room 1053, Indianapolis, IN 46202

Key Words: Rene Magritte; art; radiology; radiologists

One of the most iconic paintings of the 20th century was created by the Belgian surrealist painter Rene Magritte when he was aged 30 years. Titled The Treachery of Images (1928), it features a profile of a pipe, below which is written, “Ceci n'est pas une pipe,” French for “This is not a pipe.” Magritte's work symbolizes a number of important truths that professionals who deal regularly with images, and especially radiologists, would do well to ponder.

Magritte was born in 1898 and began drawing lessons when he was aged 12 years. His mother had a history of mental illness that prompted Magritte's father to keep her locked in her bedroom, and she ultimately committed suicide by drowning herself in the local river. Magritte studied art in Brussels, served in the military, and produced his first surrealist painting in 1926, a milestone that went largely unnoticed (1).

Magritte worked off and on in advertising, and at one point during World War II, supported himself by forging the works of other artists, such as Picasso. After the war, he also spent some time producing counterfeit bank notes to support himself. His surrealist paintings began to attain widespread recognition during the 1960s, the same decade in which he developed pancreatic cancer. He died at the age of 68 years and is buried in Brussels (1).

Magritte was frequently reproached for The Treachery of Images. Viewers complained that the image plainly was a pipe-as obvious an image of a pipe as a person could possibly imagine. Yet the

This is the author's manuscript of the article published in final edited form as:

Hackler, P. C., \& Gunderman, R. B. (2015). The Treachery of Images. Academic Radiology, 22(11), 1467-1468.

http://doi.org/10.1016/j.acra.2015.06.014 
caption explicitly denies this. Magritte's response was amusement. “The famous pipe-how people reproached me for it? And yet, could you stuff my pipe? No, it's just a representation, is it not? (1) From Magritte's point of view, the painting was just that-a painting -and to claim that he had created a pipe would have been a lie.

By entitling the painting as he does, Magritte reminds us that images-all images, whether abstract works of art, photographic portraits, or radiographs-merely represent and do not reproduce the reality they attempt to portray. In every case, what we have is not X-the thing depicted-but an image of X. Likewise, when a radiologist looks at a computed tomography (CT) image of a heart or a magnetic resonance (MR) image of a knee, what meets the eye is a representation, and never the organ or tissue itself.

This distinction is worth drawing for many reasons. For one, by including some features of reality, every image also necessarily excludes others. When we produce a chest radiograph, we choose to exclude most of the abdomen, and when we put the MR coil around the knee, we thereby choose to ignore the ankle. We cannot see everything, so we must choose to see only some things, and this means accepting the exclusion of others.

This act of framing a painting, a photograph, or a radiologic image is not a mistake, but it introduces the possibility of error. One obvious example is the so-called “corner sign,” a finding that is detectable and perhaps only partially represented on the very edge of the image. But there is a deeper potential for error. Looking at a radiologic image, we cannot exclude any anatomic abnormality except with respect to the anatomy the image in fact depicts.

A similar point applies with regard to the temporal framing of images. Most radiologic images are obtained at a particular instant in time. They show what is happening at that moment, but not necessarily what comes before or after. A transient joint subluxation may not be radiologically apparent if it happens to be reduced at the time the image is obtained. Radiologists must often rely on imagination to see any particular "snapshot" as part of an unfolding pathologic process. 
This issue of seeing only what the image includes goes deeper still, for each type of radiologic study reveals only some features of the anatomy it depicts. Plain radiography illuminates only differences in density and thickness; ultrasound illuminates only differences in acoustic impedance; and CT sees only differences in density. Fundamentally, a CT scan is nothing more than a two-dimensional collection of tiny density samples.

If the pathology in question happens to be amenable to illumination by the imaging technique in question, it will prove helpful in arriving at a diagnosis. If, however, it is invisible or opaque to that imaging technique, then it is likely to be missed. For example, differences in tissue density alone are insufficient to assess local differences in cerebral activity, and CT plays little role in functional brain imaging. So, too, ultrasound is of little use in evaluating air-filled structures.

In some cases, images not only fail to illuminate but positively obscure what they represent. For example, if a radiograph is obtained from the wrong angle, an important finding such as a fracture may not be detectable. We have seen cases in which a nondisplaced toddler fracture of the tibia was unapparent, even in retrospect, on standard frontal and lateral views. With an oblique image, however, the fracture was obvious.

Another example of a finding obscured by imaging is the failure to detect renal stones on a CT scan of the abdomen performed with intravenous contrast. The very contrast that usually helps to make pathologic process such as infection and neoplasia more conspicuous may, when it is excreted into the urine, actually hide the finding. Magritte delighted in depicting everyday realities obscured by other everyday realities, such as person's face hidden behind an apple in The Son of Man (1964).

In some cases, images actually distort the reality they portray. Magritte's work includes The Empire of Lights (1954), which depicts a house at dusk under a bright, partly cloudy sky. Likewise, CT scanning can produce considerable beam hardening artifact around metallic structures, and MR imaging can be dramatically distorted in the vicinity of ferromagnetic objects. Far from representing internal anatomy, in these cases, the imaging technique itself leads to misrepresentation. 
And finally, there are even situations in which imaging can destroy. In The Pilgrim (1966), Magritte portrays a man whose head and neck have been moved 12 inches to his right, so that they now float over his right shoulder, leaving an empty gap between his bowler hat and his shirt collar. So, too, radiologic imaging can destroy. For example, patients undergoing MR examinations have lost their lives because of the displacement of ferromagnetic objects in or about them.

Magritte was fond of saying, "Everything we see hides another thing. We always want to see what is hidden by what we see.” (1) The very existence of radiology is a reflection of this impulse, the desire to see hidden things. The integument hides what lies beneath, and radiologic techniques enable us to peer beneath the surface, to see what lies within. It is no surprise that Superman and other super heroes are portrayed as possessing the ability to see hidden things.

But when we reveal what lies within, we often lose sight of what meets the eye. When the surgeon operates on the belly, the patient's face is typically draped. Likewise, when the radiologist inspects the brain, the face of the patient is typically obscured. If a radiologist bumped into the patient whose head CT she had just been interpreting 15 minutes previously, it is likely that there would be no flash of recognition.

Radiologic imaging is frequently analogous to turning the patient inside out, making what is inside accessible to the eye but rendering what is outside no longer visible. In this sense, Magritte is exactly right: every act of representation reveals some things and hides others. Even contemporaneous trends in art, such as cubism, which attempted to reveal objects from multiple points of view at once, could only address this challenge to a limited extent (2).

A practical example of the attempt to surmount the limitations of particular imaging techniques is fusion imaging, such as positron emission tomography (PET)/CT and PET/MR imaging. Combining two different techniques, each of which is capable of revealing different biologic properties, provides a more comprehensive view of pathology-in this case, both its anatomic and functional dimensions-than either is capable of providing by itself. 
Magritte's work also helps to illuminate the essentially interpretive nature of the radiologist's work. There is a large gap between the particular pattern of photons striking the retina at any particular moment and the meaning that the radiologist extracts from the image. To take an extreme case, an experienced radiologist may instantly recognize a potentially life-threatening diagnosis such as acute pulmonary thromboembolism, whereas a complete neophyte may perceive little more than visual chaos.

The wisest radiologist is not the one who knows with $100 \%$ certainty the correct diagnosis in every case. Such a radiologist would be a fool because some cases are inherently ambiguous, and the best the radiologist can do is to provide a balanced assessment of the range of possibilities. The radiologist's mission is not to pretend to know everything, but to make full use of what can be known and present an appropriately balanced and nuanced assessment of each case.

Magritte's paintings help us to appreciate the subtleties and ambiguities involved in interpreting images, by flouting many of our cherished visual conventions. As critics have put it, Magritte is concerned with "the distance that can exist between what one thinks one knows and what one does know" (3). By showing us that what we think we know is not always the case, he reveals that interpreting images is not so straightforward as we might otherwise think (4). We operate in a milieu of uncertainty, where questioning assumptions can be one of the most fruitful exercises. 


\section{References}

1. Sylvester D. Magritte. Rev. and enl ed. Brussels: Mercatorfonds, 2009.

2. Arnason HH. History of modern art. 2nd ed. New York: Harry N Abrams, Inc, 1997.

3. Southgate TM. Rene Magritte: the telescope. In: Southgate TM, ed. The art of JAMA III: covers and essays from the Journal of the American Medical Association. New York: Oxford University Press, 2011; $180-181$.

4. Smoke and mirrors: the surreal life and work of Rene Magritte. The Independent [Internet]. 2011 June 10 [cited 2015 May 26]. Available at: http://www.independent.co.uk/arts-

entertainment/art/features/smoke-and-mirrors-thesurreal-life-and-work-of-ren-magritte-2295262.html. Accessed June 15, 2015. 